\title{
Small-cell lung cancer in the era of immunotherapy
}

\author{
Margarita Majem ${ }^{1}$, Charles M. Rudin ${ }^{2}$ \\ ${ }^{1}$ Department of Medical Oncology, Hospital de la Santa Creu i Sant Pau, Barcelona, Spain; ${ }^{2}$ Thoracic Oncology Service, Memorial Sloan Kettering \\ Cancer Center, New York, NY, USA \\ Correspondence to: Margarita Majem, MD, PhD. Department of Medical Oncology, Hospital de la Santa Creu i Sant Pau, Sant Antoni Maria Claret \\ 167, Barcelona 08025, Spain. Email: mmajem@santpau.cat. \\ Provenance: This is an invited Editorial commissioned by Guest Section Editor Dr. Qingyuan Huang, MD (Department of Thoracic Surgery, \\ Shanghai Chest Hospital, Shanghai Jiao Tong University, Shanghai, China). \\ Comment on: Ott PA, Elez E, Hiret S, et al. Pembrolizumab in Patients With Extensive-Stage Small-Cell Lung Cancer: Results From the Phase Ib \\ KEYNOTE-028 Study. J Clin Oncol 2017;35:3823-9.
}

Submitted Oct 03, 2017. Accepted for publication Oct 12, 2017.

doi: $10.21037 /$ tlcr.2017.10.06

View this article at: http://dx.doi.org/10.21037/tlcr.2017.10.06

Small-cell lung cancer represents about $13-15 \%$ of all lung cancers, accounting for more than 275,000 new cases worldwide every year (1). It is a poorly differentiated, highgrade carcinoma thought to originate from neuroendocrinecell precursors within the bronchi. Small-cell lung cancer is strongly associated with heavy tobacco exposure and typically has a high mutation burden. To date, no targeted therapy has been proven to be effective in small cell lung cancer patients $(1,2)$. Small-cell lung cancer has a high incidence of early metastasis. At diagnosis, about $70 \%$ of patients have extensive-stage disease, defined as the presence of metastatic disease by imaging or physical examination outside the hemithorax; the remaining $30 \%$ of patients have limited-stage disease, in which tumor involvement is confined to one hemithorax and can be treated in a tolerable radiation field.

Systemic therapy for patients with small-cell lung cancer has not changed substantially in several decades. In consequence, survival of patients with small-cell lung cancer has not improved, and the prognosis remains poor. The median overall survival for extensive-stage disease small-cell lung cancer patients is $9-11$ months $(2,3)$. First line treatment for patients with extensive-stage small-cell lung cancer includes a platinum doublet, generally with etoposide, with reported responses rate as high as $80 \%$. Despite this high response rate, essentially all extensivestage disease small-cell lung cancer patients will progress during or after first line treatment. Recurrent small-cell lung cancer has a particularly poor prognosis. Few therapeutic options are available for second line therapy. Topotecan, a topoisomerase inhibitor, is approved by the US Food and Drug Administration and European Union for second line treatment of recurrent small-cell lung cancer. Topotecan activity is modest, with reported overall response rates of $7-17 \%$ and median overall survivals of $6-7.8$ months $(4,5)$. Additionally, the toxicity profile of topotecan, notably hematological toxicity, may limit its use in many patients. Amrubicin, a third-generation anthracycline and potent topoisomerase II inhibitor, is approved for second line of recurrent small-cell lung cancer therapy in Japan only (6). Amrubicin achieves a median overall survival of 7.5 months, similar to that obtained with topotecan, but with higher response rate $(31.1 \%)$ and better hematological toxicity profile. There is no standard of care beyond second line therapy.

Rovalpituzumab tesirine, a DLL3-targeted antibodydrug conjugate, has recently shown promising activity in recurrent small-cell lung cancer patients with high tumor DLL3 expression ( $\geq 50 \%)$ after one or two prior lines of systemic therapy in a phase 1 clinical trial (7). Rovalpituzumab tesirine achieved a $38 \%$ confirmed objective response rate and $90 \%$ disease control rate in pretreated small-cell lung cancer patients with high DLL3 ( $\geq 50 \%)$. Of note, no DLL3-low ( $<50 \%)$ recurrent small-cell lung cancer patients had a confirmed objective response. The safety profile was manageable. A phase 3 clinical trial comparing rovalpituzumab tesirine versus topotecan in patients with recurrent DLL3-positive small-cell lung cancer is ongoing (NCT03061812), and a phase 2 study 
in patients with at least two prior lines of treatment has completed accrual (NCT02674568).

In view of these data, there is an urgent need for new approaches and better drugs for the treatment of small-cell lung cancer, including immunotherapy. Immunotherapy with checkpoint inhibitors clearly represents a promising treatment modality in several solid tumors, including lung cancer. The cytotoxic T-lymphocyte-associated protein 4 (CTLA-4) and programmed death-1 (PD-1) pathways are the most extensively studied immune checkpoint pathways. CTLA-4 and PD-1 normally attenuate immune responses and may help prevent excess immune response that would lead to autoimmunity. Tumors can co-opt these inhibitory pathways, leading to immune evasion. Blocking these key immune checkpoint pathways can reactivate immune response, potentially leading to anti-tumor responses and, conversely, to immune-mediated toxicities.

Several distinct types of checkpoint inhibitors are currently available, including inhibitory monoclonal antibodies directed against CTLA-4, PD-1, and PD-L1 (a primary ligand of PD-1 expressed on some tumors as well as immune effectors). Anti-CTLA-4 therapy is thought to act in a priming phase of the immune response, augmenting $\mathrm{T}$ cell activation in the context of tumor antigen presentation in the lymph nodes and, as a consequence, magnifying the generation of effective $\mathrm{T}$ cells that may then migrate to the tumor mass. Anti-PD-1 or PD-L1 treatments are thought to interfere primarily in the effector phase, blocking PD-L1 ligand binding to $\mathrm{PD}-1$ receptor on $\mathrm{T}$ cells in the context of the tumor microenvironment. By blocking PD-1 engagement, these treatments can promote and sustain local $\mathrm{T}$ cell activation resulting in an anti-tumor immune response (8). Concomitant inhibition of CTLA-4 pathway may further prime response to PD-1/PD-L1 inhibitors, as has been suggested in patients with advanced melanoma receiving nivolumab and ipilimumab (anti-PD-1 and antiCTLA4, respectively) (9).

Long-standing observations suggested that the immune system might play an important role in the pathophysiology of small-cell lung cancer. Small-cell lung cancer patients may present with paraneoplastic disorders, such as LambertEaton myasthenic syndrome (LEMS). Paraneoplastic neurological syndromes are thought to be immunemediated, associated with production of presumed tumor neoantigen-directed humoral antibodies that unfortunately cross-react with neural antigens (10). While LEMS can be a devastating concomitant problem, it has been noted that SCLC patients with LEMS may have a better survival outcome than those without LEMS $(11,12)$. LEMS is related to the presence of antibodies directed against voltage-gated calcium channels; the improved survival data in these patients suggests that the aberrant immune response against the nervous system might also target tumor cells through cross-reactivity to tumor antigens.

The correlation in non-small cell lung cancers between high mutation burden and higher likelihood of response to anti-PD-1 treatments (13) also supports the rationale for immunotherapy in small-cell lung cancer. Tumors with high mutation burden are inherently more likely to generate tumor-specific neoantigens- high mutation burden provides an increased substrate of targets that may trigger an immune response through their presentation to T cells. Small-cell lung cancer genomes are notable for high mutation burdens and genomic instability, providing ample opportunity for development of tumor-specific antigen generation.

The immune checkpoint inhibitor antibodies antiCLTA-4, anti-PD-1 and anti-PD-L1 and have significantly improved overall survival in several solid tumors, including non-small cell lung cancer. To date, little evidence about the efficacy of immunotherapy in small-cell lung cancer has been published. The CTLA-4 inhibitor ipilimumab failed to demonstrate an overall survival benefit in combination with etoposide and platinum in first line setting for patients with extensive-stage small-cell lung cancer in the CA184156 phase 3 trial (14). Anti-CTLA-4 plus chemotherapy is a strategy that is unlikely to be further explored.

The phase $1 / 2$ CheckMate 032 evaluates nivolumab \pm ipilimumab in different tumor types including recurrent small-cell lung cancer. The first report of the smallcell lung cancer cohort included 216 patients allocated in three different schedules: nivolumab alone or in combination with ipilimumab at two different doses. Anti-tumour activity in small-cell lung cancer patients was seen in all schedules (15). Confirmed responses were observed in $10 \%$ (95\% CI: $5-18$ ) of patients with nivolumab $3 \mathrm{mg} / \mathrm{kg}, 23 \%$ (13-36\%) with nivolumab $1 \mathrm{mg} / \mathrm{kg}$ plus ipilimumab $3 \mathrm{mg} / \mathrm{kg}$, and $19 \%(9-31 \%)$ with nivolumab $3 \mathrm{mg} / \mathrm{kg}$ plus ipilimumab $1 \mathrm{mg} / \mathrm{kg}$. The median overall survival was 4.4 (95\% CI: 3.0-9.3) months in the nivolumab cohort, 7.7 (95\% CI: 3.6-18.0) months in the nivolumab $1 \mathrm{mg} / \mathrm{kg}$ plus ipilimumab $3 \mathrm{mg} / \mathrm{kg}$ cohort, and 6.0 (95\% CI: 3.6-11.0) months in the nivolumab $3 \mathrm{mg} / \mathrm{kg}$ plus ipilimumab $1 \mathrm{mg} / \mathrm{kg}$ cohort. A pre-planned exploratory analysis showed that PD-L1 expression did not correlate with tumor response, and seemed not to be useful for treatment decision-making. A randomized cohort was added later to evaluate nivolumab $3 \mathrm{mg} / \mathrm{kg} \pm$ ipilimumab $1 \mathrm{mg} / \mathrm{kg}$ (16). Response rates were $12 \%$ (7-18\%) for 
nivolumab and $21 \%$ (13-31\%) for ipilimumab + nivolumab. Grade 3/4 treatment related adverse events and deaths were more common with nivolumab + ipilimumab than with nivolumab as previously seen in other tumor types.

Results of pembrolizumab in patients with pretreated extensive-stage small-cell lung cancer have been recently published by Ott and colleagues (17). The Keynote-028 trial is an open label phase Ib multi-cohort (20 cohorts) study that includes patients with PD-L1 positive tumors (membranous PD-L1 expression in $\geq 1 \%$ of cells in tumor and associated inflammatory cells or positive staining in stroma). The pretreated small-cell lung cancer cohort screened 163 patients, of which 145 had adequate samples for PD-L1 analysis. PD-L1 expression was positive in 46 patients (31.7\%), and finally 24 patients were treated. They received pembrolizumab at $10 \mathrm{mg} / \mathrm{kg}$ every 2 weeks up to a maximum of 24 months. Pembrolizumab achieved an overall response rate of $33 \%$ (16-65\%), with a median duration of response of 19.4 months ( $\geq 3.6$ to $\geq 20$ months), and a median overall survival of 9.7 (95\% CI: 4.7-NR) months. The safety profile of pembrolizumab was consistent with that reported in other solid tumors, being asthenia and fatigue the most common side effects.

The PD-L1 pathway participates in immune suppression in the tumor microenvironment. The PD-L1 ligand is being investigated as a predictive biomarker for anti-PD-1 and anti-PD-L1 therapies. In non-small cell lung cancer, a positive correlation has been observed between response rate to anti-PD-1 and anti-PD-L1 therapies and PD-L1 expression in several tumor types including non-small cell lung cancer. However, it is important to underline that not all patients with PD-L1-positive tumors will respond, and conversely, patients with PD-L1-negative tumors may respond. As a consequence, the role of PD-L1 expression to select patients for anti-PD-1 and anti-PD-L1 therapies remains controversial in the majority of tumor types.

Reports about PD-L1 expression in small-cell lung cancer tumors are contradictory, although it seems to be lower than in non-small cell lung cancer tumors. Reported PD-L1 expression in retrospective studies in small-cell lung cancer varies widely, from a few percentage points to over $70 \%(18,19)$. In the Keynote- 028 trial, a reported $31.7 \%$ of small-cell lung cancer patients tested for PD-L1 expression were positive, whereas only $17 \%$ of the analyzed patients in the CheckMate 032 had a PD-L1 expression $\geq 1 \%(15,17)$.

Although the Keynote 028 trial reported a response rate and a median overall survival in pretreated small-cell lung cancer patients expressing PD-L1 that were numerically higher than those reported in the CheckMate 032 trial with nivolumab +/- ipilimumab in small-cell lung cancer patients not selected by PD-L1 expression, we would caution against making any conclusions in this cross-trial comparison. Notably in the CheckMate 032 trial an essentially equal likelihood of responses was observed in PD-L1 negative and PD-L1 positive small-cell lung cancer tumors, not only in the nivolumab cohort but also in the combination cohort, suggesting that PD-L1 does not enrich for response in small-cell lung cancer (15). Whether PD-L1 expression is of any predictive utility in small-cell lung cancer must await analysis in a larger population.

Several phase 3 clinical trials of immunotherapy in extensive-stage small-cell lung cancer are currently ongoing not only as monotherapy but also in combination with chemotherapy or with other therapies. Nivolumab is being evaluated in second line versus chemotherapy (CheckMate 331, NCT02481830) and also as maintenance therapy after first line treatment alone or in combination with ipilimumab versus placebo (CheckMate 451, NCT02538666). Pembrolizumab is being evaluated in combination with chemotherapy in first line versus chemotherapy alone (KEYNOTE-604, NCT03066778). Durvalumab with or without Tremelimumab is being evaluated in combination with chemotherapy in first line versus chemotherapy alone (Caspian, NCT03043872). Atezolizumab is also being evaluated in combination with chemotherapy in first line versus chemotherapy alone (IMpower133-NCT02763579). None of these trials is selecting patients based on PD-L1 expression, although tumor samples are collected in order to perform exploratory biomarker analysis.

Results of these definitive phase 3 studies are eagerly awaited. The initial data with nivolumab, nivolumab plus ipilimumab, and now pembrolizumab are strongly encouraging, suggesting not only that immunotherapy can work in this disease, but that some of the resulting responses have impressive durability. The results of large randomized trials projected to complete enrollment in 2018 may at long last change the paradigm of treatment for patients with small-cell lung cancer.

\section{Acknowledgements}

None.

\section{Footnote}

Conflicts of Interest: The authors have no conflicts of interest to declare. 


\section{References}

1. Ferlay J, Steliarova-Foucher E, Lortet-Tieulent J, et al. Cancer incidence and mortality patterns in Europe: estimates for 40 countries in 2012. Eur J Cancer 2013;49:1374-403.

2. Fruh M, De Ruysscher D, Popat S, et al. Small-cell lung cancer (SCLC): ESMO Clinical Practice Guidelines for diagnosis, treatment and follow-up. Ann Oncol 2013;24 Suppl 6:vi99-105.

3. National Comprehensive Cancer Network. NCCN Clinical Prctice Guidelines in Oncology: small cell lung cancer, version 1.2018.

4. Horita N, Yamamoto M, Sato T, et al. Topotecan for Relapsed Small-cell Lung Cancer: Systematic Review and Meta-Analysis of 1347 Patients. Sci Rep 2015;5:15437.

5. O'Brien ME, Ciuleanu TE, Tsekov H, et al. Phase III trial comparing supportive care alone with supportive care with oral topotecan in patients with relapsed small-cell lung cancer. J Clin Oncol 2006;24:5441-7.

6. von Pawel J, Jotte R, Spigel DR, et al. Randomized phase III trial of amrubicin versus topotecan as second-line treatment for patients with small-cell lung cancer. J Clin Oncol 2014;32:4012-9.

7. Rudin CM, Pietanza MC, Bauer TM, et al. Rovalpituzumab tesirine, a DLL3-targeted antibody-drug conjugate, in recurrent small-cell lung cancer: a first-inhuman, first-in-class, open-label, phase 1 study. Lancet Oncol 2017;18:42-51.

8. Ribas A. Tumor immunotherapy directed at PD-1. N Engl J Med 2012;366:2517-9.

9. Wolchok JD, Chiarion-Sileni V, Gonzalez R, et al. Overall Survival with Combined Nivolumab and Ipilimumab in Advanced Melanoma. N Engl J Med 2017;377:1345-56.

10. Darnell RB, Posner JB. Paraneoplastic syndromes affecting the nervous system. Semin Oncol 2006;33:270-98.

11. Maddison P, Newsom-Davis J, Mills KR, et al. Favourable prognosis in Lambert-Eaton myasthenic syndrome and small-cell lung carcinoma. Lancet 1999;353:117-8.

12. Maddison P, Lang B. Paraneoplastic neurological autoimmunity and survival in small-cell lung cancer. J Neuroimmunol 2008;201-202:159-62.

13. Rizvi NA, Hellmann MD, Snyder A, et al. Cancer immunology. Mutational landscape determines sensitivity to PD-1 blockade in non-small cell lung cancer. Science 2015;348:124-8.

14. Reck M, Luft A, Szczesna A, et al. Phase III Randomized Trial of Ipilimumab Plus Etoposide and Platinum Versus Placebo Plus Etoposide and Platinum in Extensive-Stage Small-Cell Lung Cancer. J Clin Oncol 2016;34:3740-8.

15. Antonia SJ, Lopez-Martin JA, Bendell J, et al. Nivolumab alone and nivolumab plus ipilimumab in recurrent smallcell lung cancer (CheckMate 032): a multicentre, openlabel, phase 1/2 trial. Lancet Oncol 2016;17:883-95.

16. Hellmann MD, Ott PA, Zugazagoitia J. Nivolumab (nivo) \pm ipilimumab (ipi) in advanced small-cell lung cancer (SCLC): First report of a randomized expansion cohort from CheckMate 032. J Clin Oncol 2017;35:abstr 8503.

17. Ott PA, Elez E, Hiret S, et al. Pembrolizumab in Patients With Extensive-Stage Small-Cell Lung Cancer: Results From the Phase Ib KEYNOTE-028 Study. J Clin Oncol 2017;35:3823-9.

18. Schultheis AM, Scheel AH, Ozretic L, et al. PD-L1 expression in small cell neuroendocrine carcinomas. Eur J Cancer 2015;51:421-6.

19. Ishii H, Azuma K, Kawahara A, et al. Significance of programmed cell death-ligand 1 expression and its association with survival in patients with small cell lung cancer. J Thorac Oncol 2015;10:426-30.
Cite this article as: Majem M, Rudin CM. Small-cell lung cancer in the era of immunotherapy. Transl Lung Cancer Res 2017;6(Suppl 1):S67-S70. doi: 10.21037/tlcr.2017.10.06 